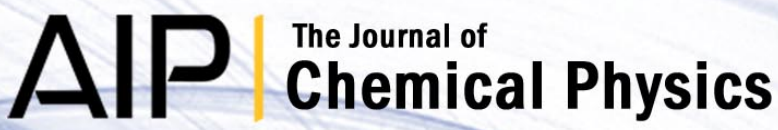

\section{Mesoscale hydrodynamic modeling of a colloid in shear-thinning viscoelastic fluids under shear flow}

Shichen Ji, Run Jiang, Roland G. Winkler, and Gerhard Gompper

Citation: J. Chem. Phys. 135, 134116 (2011); doi: 10.1063/1.3646307

View online: http://dx.doi.org/10.1063/1.3646307

View Table of Contents: http://jcp.aip.org/resource/1/JCPSA6/v135/i13

Published by the American Institute of Physics.

\section{Additional information on J. Chem. Phys.}

Journal Homepage: http://jcp.aip.org/

Journal Information: http://jcp.aip.org/about/about_the_journal

Top downloads: http://jcp.aip.org/features/most_downloaded

Information for Authors: http://jcp.aip.org/authors

\section{ADVERTISEMENT}

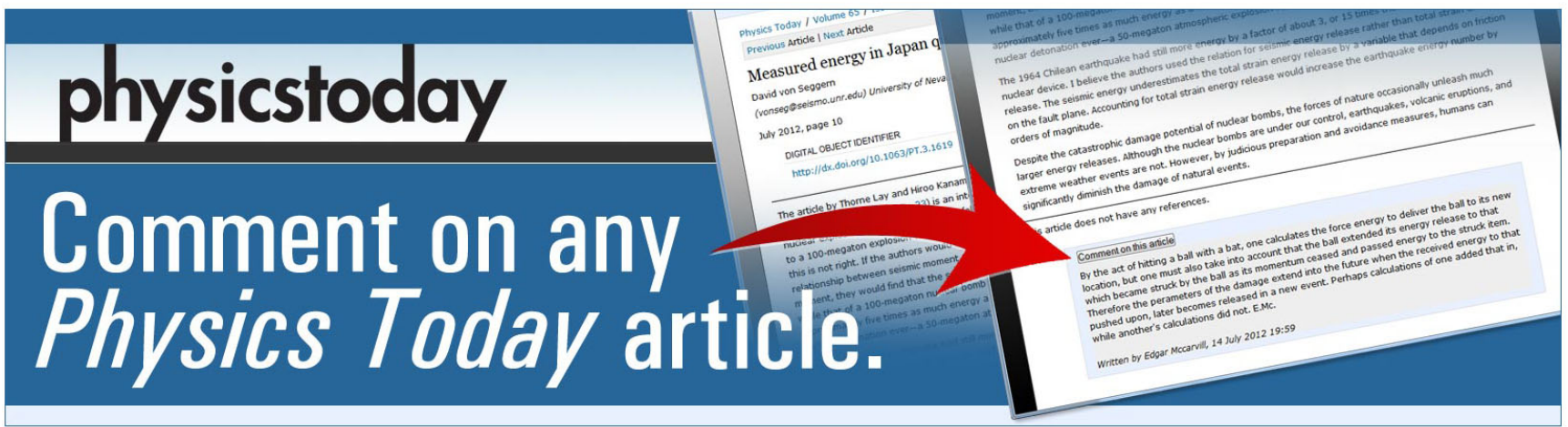




\title{
Mesoscale hydrodynamic modeling of a colloid in shear-thinning viscoelastic fluids under shear flow
}

\author{
Shichen Ji, Run Jiang, Roland G. Winkler, and Gerhard Gompper ${ }^{a)}$ \\ Theoretical Soft Matter and Biophysics, Institute of Complex Systems and Institute for Advanced Simulation, \\ Forschungszentrum Jülich, D-52425 Jülich, Germany
}

(Received 13 July 2011; accepted 15 September 2011; published online 6 October 2011)

\begin{abstract}
In order to study the dynamics of colloidal suspensions with viscoelastic solvents, a simple mesoscopic model of the solvent is required. We propose to extend the multiparticle collision dynamics (MPC) technique - a particle-based simulation method, which has been successfully applied to study the hydrodynamic behavior of many complex fluids with Newtonian solvent-to shear-thinning viscoelastic solvents. Here, the normal MPC particles are replaced by dumbbells with finite-extensible nonlinear elastic (FENE) springs. We have studied the properties of FENE-dumbbell fluids under simple shear flow with shear rate $\dot{\gamma}$. The stress tensor is calculated, and the viscosity $\eta$ and the first normal-stress coefficient $\Psi_{1}$ are obtained. Shear-thinning behavior is found for reduced shear rates $\Gamma=\dot{\gamma} \tau>1$, where $\tau$ is a characteristic dumbbell relaxation time. Here, both $\eta$ and $\Psi_{1}$ display power-law behavior in the shear-thinning regime. Thus, the FENE-dumbbell fluid with MPC collisions provides a good description of viscoelastic fluids. As a first application, we study the flow behavior of a colloid in a shear-thinning viscoelastic fluid in two dimensions. A slowing down of the colloid rotation in a viscoelastic fluid compared to a Newtonian fluid is obtained, in agreement with recent numerical calculations and experimental results. (C) 2011 American Institute of Physics. [doi:10.1063/1.3646307]
\end{abstract}

\section{INTRODUCTION}

During the last decades, the research areas of colloids, polymers, membranes, and liquid crystals, which were previously largely independent, have been integrated into the single field of soft matter. Their common property is high sensitivity to external stimuli, which suggests that thermal fluctuations play a major role in such systems. Under non-equilibrium conditions, soft matter systems exhibit both viscous and elastic characteristics-they are viscoelastic. ${ }^{1}$ Prominent examples for such a behavior are polymer melts and solutions. ${ }^{2,3}$

The properties of soft matter systems are mainly determined by their mesoscopic structure. Due to the large separation of length and time scales between the atomic scale of the solvent and the mesoscale of the solute, the simulation of the dynamical behavior of soft matter is particularly challenging. Traditional molecular dynamics simulations are often inappropriate because of the unaffordable simulation time, especially when hydrodynamics needs to be taken into account. For a colloidal suspension in a viscoelastic solvent, e.g., proteins in a cell, or spherical or rod-like colloids dispersed in polymer solutions, ${ }^{4-7}$ the situation is even more complex. The solvent itself is now a complex fluid too. To be able to address and unravel dynamical and rheological properties of such complex systems requires a simplified mesoscopic model of a viscoelastic solvent, preferentially in combination with a mesoscopic hydrodynamic simulation technique, such as the multiparticle collision dynamics (MPC) approach. ${ }^{8,9}$

\footnotetext{
a) Author to whom correspondence should be addressed. Electronic mail: g.gompper@fz-juelich.de.
}

The MPC technique is a particle-based simulation method with a high computational efficiency, which was recently introduced by Malevanets and Kapral. ${ }^{10}$ The MPC algorithm consists of alternating streaming and collision steps. Both, thermal fluctuations and hydrodynamic interactions are inherently included in the MPC approach. MPC has been shown to describe the dynamics of Newtonian fluids very well, and has been successfully applied to the study of the hydrodynamic behavior of various complex fluids. 8,9

In this article, we propose an extension of the MPC technique to shear-thinning viscoelastic fluids by replacing the point particles of simple MPC with dumbbells of finite extensibility. A dumbbell is a maximally coarse-grained but very effective model of a polymer, as the dumbbell can orient and stretch-reflecting the entropic properties of a polymer-under non-equilibrium conditions. ${ }^{3,11,12}$ The MPC approach has been used previously to study the flow behavior of harmonic dumbbells. ${ }^{13}$ As has been shown, a fluid consisting of harmonic dumbbells only captures the viscoelastic behavior of a Maxwell fluid well. ${ }^{13}$ The main advantage of harmonic dumbbells is that the integration of the equations of motions in the streaming step can be performed analytically, hence, the full efficiency of the MPC approach for point particles is conserved. Although the shear-induced orientation and elongation in shear flow are well reproduced, the shear-thinning behavior cannot be captured naturally with harmonic dumbbells. However, various studies have shown that the shear-thinning behavior of viscoelastic fluids can be well reproduced by finite-extensible nonlinear elastic (FENE) dumbbells in solution. ${ }^{3,12}$ A similar idea to employ FENE dumbbells as a mesoscale model for viscoelastic fluids in 
dissipative particle dynamics (DPD) simulation was pursued in Ref. 14. The simulations show a non-zero normal stress difference, but only very weak shear-thinning behavior. This DPD model was recently extended to mixtures of FENE dumbbells and standard DPD particles. ${ }^{15}$

The article is organized as follows. In Sec. II, the FENE dumbbell fluid in combination with MPC is introduced. Properties of this fluid in simple shear flow are investigated in Sec. III, in particular, the orientation, stretching, and tumbling of individual dumbbells (see Sec. III A) and the rheology of the dumbbell fluid (see Sec. III B), as a function of shear rate. This model for a shear-thinning viscoelastic fluid is then employed in Sec. IV to study the rotation of a colloidal particle in shear flow. The results are summarized in Sec. V.

\section{MODEL AND METHOD}

\section{A. Multiparticle collision dynamics}

A simple MPC fluid comprises $N$ point particles of mass $m$, which are characterized by their positions $\boldsymbol{r}_{i}$ and velocities $\boldsymbol{v}_{i}$, with $i=1, \ldots, N$. In two dimensions, the particles are distributed randomly within a simulation box of size $L_{x} \times L_{y}$ with number density $\rho=N /\left(L_{x} L_{y}\right)$. Their dynamics consists of alternating streaming and collision steps. In the streaming step, the particles move ballistically and their positions evolve according to

$$
\boldsymbol{r}_{i}(t+h)=\boldsymbol{r}_{i}(t)+\boldsymbol{v}_{i}(t) h
$$

during a time interval $h$, which is called the collision time. In the collision step, fluid particles exchange momentum locally by a stochastic process. This is achieved by first sorting all particles into the cells of a square lattice with lattice constant $a$. Then, the particles within a cell interact with each other simultaneously such that mass and momentum are conserved. Different collision rules have been suggested, which, in addition, either conserve energy ${ }^{10}$ (a microcanonical approach called stochastic rotation dynamics) or keep the temperature constant $^{16,17}$ (a canonical approach called Andersen thermostat (MPC-AT)). The MPC-AT approach has the additional advantage that it can be extended to conserve angular momentum in each cell during the collision step, a feature which has been shown to be relevant for studies of rotating droplets and colloids. ${ }^{18}$ Since we are interested in the behavior of rotating colloids, see Sec. IV, we employ here an Andersen thermostat with angular momentum conservation (MPC-AT $+a$ ), where the velocity of particle $i$ after the collision is given by ${ }^{18}$

$$
\begin{aligned}
\boldsymbol{v}_{i}(t+h)= & \boldsymbol{v}_{c}+\boldsymbol{v}_{i}^{r a n}-\sum_{j \in \text { cell }} \boldsymbol{v}_{j}^{\text {ran }} / N_{c} \\
& +m \boldsymbol{\Pi}^{-1} \sum_{j \in \text { cell }}\left\{\boldsymbol{r}_{j c} \times\left(\boldsymbol{v}_{j}-\boldsymbol{v}_{j}^{r a n}\right)\right\} \times \boldsymbol{r}_{i c} .
\end{aligned}
$$

Here, $N_{c}$ is the number of particles in a cell, $\boldsymbol{v}_{c}$ and $\boldsymbol{r}_{c}$ are the velocity and position of the center of mass of all particles in a cell, respectively, $\boldsymbol{r}_{i c}=\boldsymbol{r}_{i}-\boldsymbol{r}_{c}$ is the position of particle $i$ relative to the center of mass of the cell, and $\Pi$ is the moment-of-inertia tensor of the particles in the cell. Finally, the velocities $\boldsymbol{v}_{i}^{r a n}$ are chosen from a Maxwell-Boltzmann distribution with temperature $T$. To ensure Galilean invariance, a random shift of the lattice is applied before every collision step. $^{19,20}$

An important feature of MPC is that the dynamics is well defined for any choice of collision time, and transport coefficients and viscosity can be well tuned by controlling the collision time. ${ }^{8,9}$ In particular, the regime of low Reynolds numbers, characteristic for most soft-matter systems, is obtained for small collision times, so that the mean free path is much smaller than the cell size $a .^{21,22}$ Analytical expressions for the viscosity and other transport coefficients for the MPC-AT $+a$ method have been derived in Ref. 23.

\section{B. FENE Dumbbells}

To extend the MPC method to simulations of viscoelastic solvents, we augment the simple MPC method by connecting two MPC particles by the FENE potential: ${ }^{3}$

$$
U(R)=-\frac{k}{2} R_{0}^{2} \ln \left[1-\left(\frac{R}{R_{0}}\right)^{2}\right] .
$$

Here, $\boldsymbol{R}$ is the bond vector between the two particles of a dumbbell, $R=|\boldsymbol{R}|, R_{0}$ is the maximum allowed elongation, and $k$ is the spring constant. Usually, $b=k R_{0}^{2}$ is defined as the FENE parameter. ${ }^{3}$ The dynamics of the FENE dumbbells can be simply integrated into a MPC simulation. During the streaming step, the motion of each dumbbell is updated according to the velocity Verlet algorithm with a time interval $\Delta t_{d}$. Here, it is important to emphasize that each dumbbell moves independently from all others during the streaming step. Then, in the collision steps, the two point particles of a dumbbell interact with other dumbbell particles as simple MPC particles, i.e., in the collision step, the FENE potential plays no role, and all particles take part in the collision step in the same way as in a simple MPC fluid. The same approach has been used in Ref. 13 for harmonic dumbbells. As there are only collisional interactions between dumbbells in the simulation, the system corresponds to a dilute polymer solution.

\section{Shear flow and stress tensor}

Lees-Edwards boundary conditions ${ }^{24}$ are applied to impose simple shear flow, with flow in the $x$ direction and a shear gradient in the $y$ direction, with the shear rate $\dot{\gamma}=d v_{x} / d y$. The calculation of rheological properties is based on the system's stress tensor $\sigma_{\alpha \beta}$ (where $\alpha$ and $\beta$ are the Cartesian coordinates). Recently, expressions for the stress tensor $\sigma_{\alpha \beta}$ of a MPC fluid have been derived in Ref. 25, both in thermal equilibrium and under shear flow conditions. For the FENEdumbbell fluid, this approach yields

$$
\begin{aligned}
\sigma_{\alpha \beta}= & -\frac{1}{V} \sum_{i=1}^{N} m\left\langle\left(v_{i, \alpha}-\bar{v}_{\alpha}\right)\left(v_{i \beta}-\bar{v}_{\beta}\right)\right\rangle_{t} \\
& -\frac{1}{V h} \sum_{i=1}^{N}\left\langle\Delta p_{i, \alpha} r_{i, \beta}\right\rangle_{t}-\frac{1}{V} \sum_{j=1}^{N / 2}\left\langle F_{j, \alpha} R_{j, \beta}\right\rangle_{t},
\end{aligned}
$$


where $\langle\cdots\rangle_{t}$ denotes time average, $V$ is the volume of the simulation box, and $\overline{\boldsymbol{v}}=\dot{\gamma} y \boldsymbol{e}_{x}$ is the ideal velocity of an imposed shear-flow field. $\Delta \boldsymbol{p}_{i}$ is the change in momentum of particle $i$ in a collision, which is given by

$$
\Delta \boldsymbol{p}_{i}=m\left(\boldsymbol{v}_{i}-\hat{\boldsymbol{v}}_{i}\right),
$$

where $\hat{\boldsymbol{v}}_{i}$ and $\boldsymbol{v}_{i}$ are the velocities of particle $i$ before and after the collision process, respectively. The last term accounts for the contribution of the bonds of the dumbbells, where $\boldsymbol{F}_{j}$ is the force within dumbbell $j$ (pointing in the same direction as the bond vector $\mathbf{R}_{j}$ ). Alternative routes to calculate the stress tensor of a MPC fluid have been proposed in Refs. 26 and 27.

Once the stress tensor is known, the shear viscosity $\eta$ and the first normal-stress coefficient $\Psi_{1}$ can be calculated as

$$
\begin{gathered}
\eta=\sigma_{x y} / \dot{\gamma}, \\
\Psi_{1}=\left(\sigma_{x x}-\sigma_{y y}\right) / \dot{\gamma}^{2} .
\end{gathered}
$$

We will present our result as a reduced viscosity, which is defined as

$$
\eta_{r}=\frac{\eta-\eta_{s}}{\eta_{0}-\eta_{s}}
$$

where $\eta_{0}$ is the zero-shear viscosity of the system and $\eta_{s}$ is the viscosity of a simple MPC solvent. For the MPC-AT $+a$ version of MPC in two dimensions, the analytical result ${ }^{17,23}$

$$
\eta_{s}=\rho\left[\frac{h k_{B} T}{m}\left(\frac{\rho}{\rho-1}-\frac{1}{2}\right)+\frac{a^{2}}{24 h} \frac{\rho-7 / 5}{\rho}\right]
$$

has been derived, where $k_{B}$ is the Boltzmann constant.

\section{Simulation parameters}

In the remainder of this paper, the simulation data are displayed in reduced units, with lengths measured in units of the lattice constant $a$, time in units of $\tau_{0}=a \sqrt{m / k_{B} T}$, and shear rates in units of $1 / \tau_{0}$. This corresponds to the parameter choice $m=1, a=1$, and $k_{B} T=1$. In these units, the mean free path of MPC point particles (without FENE interactions) equals the collision time $h$. If not mentioned otherwise, we employ the following set of parameters. The average number density of MPC particle is set to $\rho=10$ and the collision time is set to $h=0.02$. Such a small $h$ ensures that the system is in a fluid-like region with low Reynolds number. ${ }^{21}$ The spring constant of the FENE dumbbells is taken to be $k=0.2$ and their maximum extension $R_{0}=6.32$, which corresponds to $b=8$. The time interval to update FENE dumbbells is set as $\Delta t_{d}=0.002$. On very rare occasions, if overextension occurs (for the highest shear rate considered, $\dot{\gamma}=0.2$, this happens with a probability of $5 \times 10^{-6}$ ), the dumbbell is replaced by a new one with an elongation slightly smaller than the maximum extension. The system size is $L_{x}=L_{y}=50$ if not stated otherwise.

Shear flow is characterized by the dimensionless shear rate (or Weissenberg number) $\Gamma=\dot{\gamma} \tau$, where the characteristic time $\tau$ of a harmonic dumbbell is defined as $\tau=\zeta /(4 k)$, in analogy to Ref. 3, with $\zeta$ being the friction coefficient. Using the Einstein relation $\zeta=k_{B} T / D$, where $D$ is the monomer

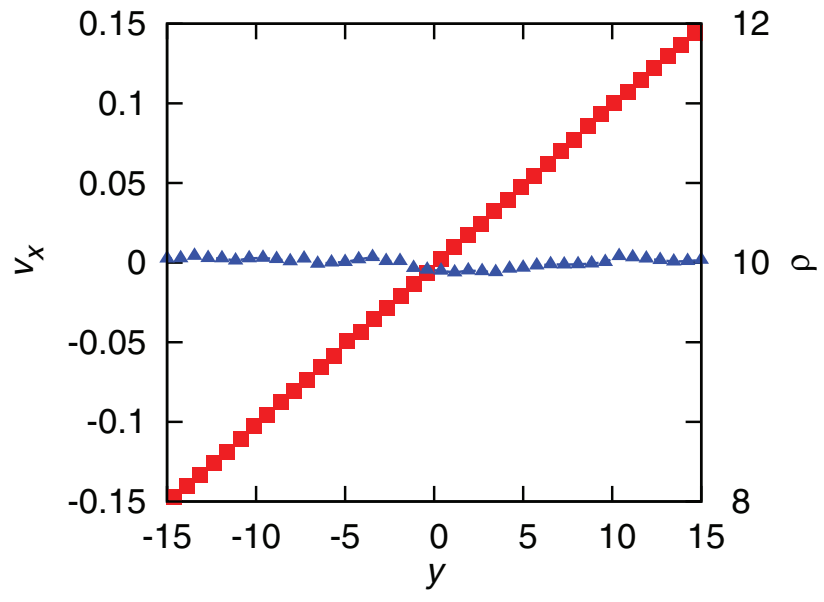

FIG. 1. Velocity $(\boldsymbol{\square})$ and density $(\boldsymbol{\Delta})$ profiles in simple shear flow as a function of position $y$ in the gradient direction. The shear rate is $\dot{\gamma}=0.01$, the density of MPC particles is $\rho=10$, and the collision time is $h=0.02$. The spring constant of the FENE dumbbell is $k=0.2$, and the FENE parameter is $b=8$.

diffusion coefficient, we find ${ }^{13}$

$$
\tau=k_{B} T /(4 D k)
$$

For $D$, we use the diffusion coefficient of a point particle in a simple MPC-AT $+a$ fluid, which in $d$ dimensions is given by $^{23}$

$$
D=\frac{k_{B} T h}{m}\left(\frac{\rho}{\rho-(d+1) / 2}-\frac{1}{2}\right)
$$

for sufficiently large $\rho$. For the standard parameters given above, we obtain $\tau=92 \tau_{0}$. Note that $\tau$ is half of the dumbbell bond-vector relaxation time.

\section{FENE-DUMBBELL FLUID IN SIMPLE SHEAR FLOW}

\section{A. Dumbbell orientation, stretching, and tumbling}

The velocity and density profiles of a FENE-dumbbell fluid under shear flow are shown in Fig. 1. A linear velocity profile is obtained, as expected. The density is constant, which demonstrates that the homogeneous distribution of the MPC particles is not altered by the shear-flow field.

We first investigate the conformational and dynamical properties of an individual dumbbell in a FENE-dumbbell fluid under shear flow. As illustrated in Fig. 2 by density plots, the dumbbell orientation and stretching increases with increasing shear rate. For a low shear rate of $\Gamma=0.092$, the distribution of the end-points is essentially isotropic and cannot be distinguished easily from the equilibrium distribution. For the intermediate shear rate $\Gamma=0.92$, the distribution function exhibits a preferred direction, with an orientation angle of about $30^{\circ}$ with respect to the flow direction, comparable to a harmonic dumbbell. ${ }^{13}$ Finally, for the high shear rate $\Gamma=9.2$, the dumbbell strongly aligns with the flow direction and the finite extensibility limits further stretching.

This behavior can be made more quantitative by calculating the average dumbbell orientation and the average endto-end distance $R$. As shown in Fig. 3, the dumbbell extension is almost independent of the shear rate for $\Gamma \leq 0.5$. For larger shear rates, $R$ increases and gradually saturates in the 

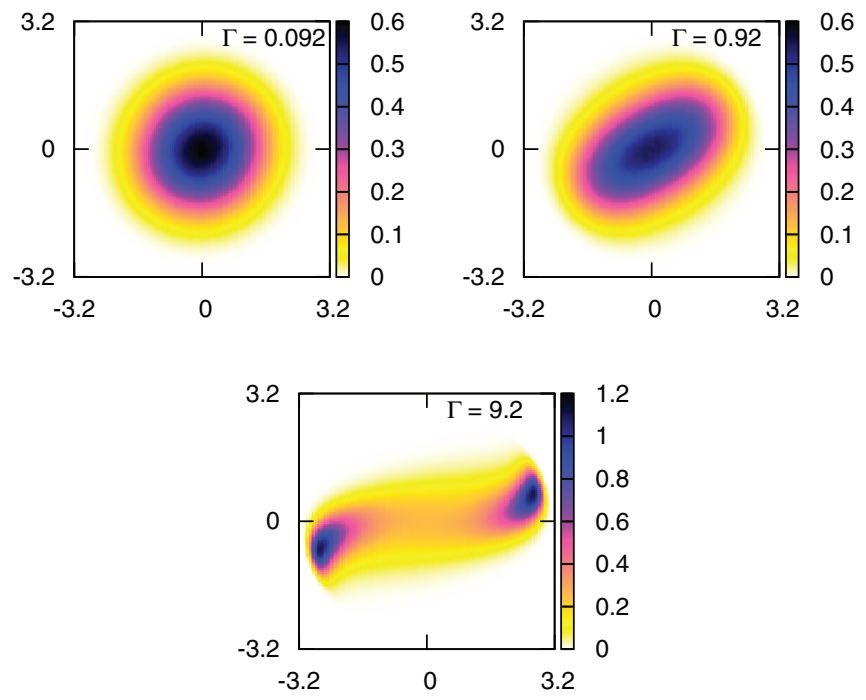

FIG. 2. Density distribution of dumbbell monomers relative to the dumbbell's center of mass (arb. units) for the indicated reduced shear rates $\Gamma=\dot{\gamma} \tau$. The other parameters are the same as in Fig. 1. The maximum extension is $R_{0}=\sqrt{b / k}=6.325$. The integrated probability is the same in all three plots.

asymptotic limit due to the FENE potential. Simultaneously, a shear-induced alignment occurs, which we characterize by the inclination angle $\theta$ determined by

$$
\tan (2 \theta)=\frac{2\left\langle G_{x y}\right\rangle}{\left\langle G_{x x}\right\rangle-\left\langle G_{y y}\right\rangle},
$$

i.e., $\theta$ is the angle between the major axis of the radius-ofgyration tensor $G_{\alpha \beta}=R_{\alpha} R_{\beta} / 4$, with Cartesian coordinated $\alpha, \beta$, and the flow direction. In the weak-flow regime, $\theta$ approaches the equilibrium value of $45^{\circ}$ for $\Gamma \rightarrow 0$, and $\tan (2 \theta)$ shows a power-law behavior

$$
\tan (2 \theta) \sim \Gamma^{-1} \text { for } \Gamma<1,
$$

in agreement with theoretical predictions. ${ }^{28}$ At shear rates $\Gamma>1, \tan (2 \theta)$ is expected to exhibit a crossover to the asymptotic power-law decay ${ }^{28}$

$$
\tan (2 \theta) \sim \Gamma^{-1 / 3} \text { for } \Gamma \gg 1
$$

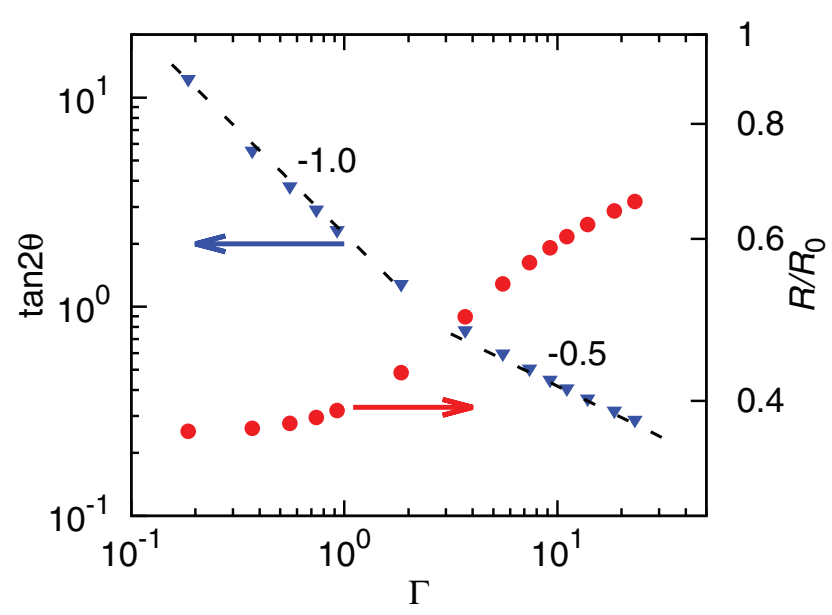

FIG. 3. Extension $R / R_{0}(\bullet)$ and inclination $\tan (2 \theta)(\boldsymbol{\nabla})$ as a function of the reduced shear rate $\Gamma$. The other parameters are the same as those in Fig. 1. The numbers indicate the exponents of power-law regimes.

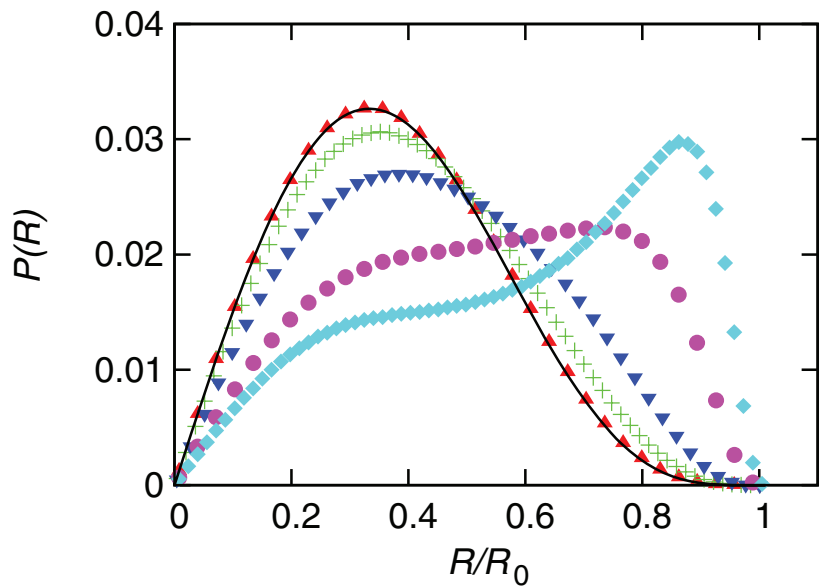

FIG. 4. Probability distribution of the dumbbell extension for various shear rates $\Gamma=0(\boldsymbol{\Delta}), 0.92(+), 1.84(\boldsymbol{\nabla}), 4.6(\bullet)$, and $9.2(\bullet)$. The solid line is obtained from the Boltzmann factor with the FENE potential. The other parameters are the same as in Fig. 1.

The simulation data in Fig. 3 for the largest investigated shear rates are in the crossover regime, and follow an effective power law $\Gamma^{-0.5}$.

It is also interesting to consider the effect of shear flow on the end-to-end distance distribution of the dumbbells, which is displayed in Fig. 4. At thermal equilibrium, finiteextensibility effects are weak and the distribution is very well described by the Gaussian distribution of a harmonic dumbbell. The peak at $R / R_{0}<0.5$ switches to a peak at $R / R_{0}$ $>0.5$ when $\Gamma \simeq 4$. Simultaneously, the maximum extensibility becomes relevant. However, a shoulder in the distribution functions near the equilibrium extension $R_{e q}$ remains prominent even for higher shear rates.

Our simulations also provide information about the dynamical behavior of dumbbells under shear flow. It is well known that polymers exhibit a tumbling motion in shear flow. ${ }^{29-36}$ Here, we discuss the rotational dynamics in terms of the average rotation frequency $\omega$ of a dumbbell, which is calculated as

$$
\langle\omega\rangle_{t}=\langle L / I\rangle_{t},
$$

where $I=m R^{2} / 2$ is the moment of inertia and $L$ $=m\left(R_{x} d R_{y} / d t-R_{y} d R_{x} / d t\right)$ is the angular momentum. ${ }^{37}$ The results are displayed in Fig. 5. At low shear rates, the relation $\omega=\dot{\gamma} / 2$ applies, ${ }^{38}$ as expected. With increasing shear rate, $\omega / \dot{\gamma}$ decreases and reaches a power-law regime

$$
\omega / \dot{\gamma} \sim \Gamma^{-0.5} .
$$

A similar scaling behavior has been found in the nonequilibrium molecular dynamics simulations of polymer chains in Ref. 37.

\section{B. Rheology of FENE-dumbbell fluids}

We calculate the reduced viscosity $\eta_{r}$ of FENE dumbbell fluids with Eqs. (8) and (9). The results are shown in Fig. 6 as a function of the reduced shear rate. The reduced viscosity remains constant in the weak flow regime, $\Gamma \leq 1$. In this regime, the viscosity $\eta$ itself is well described by the 


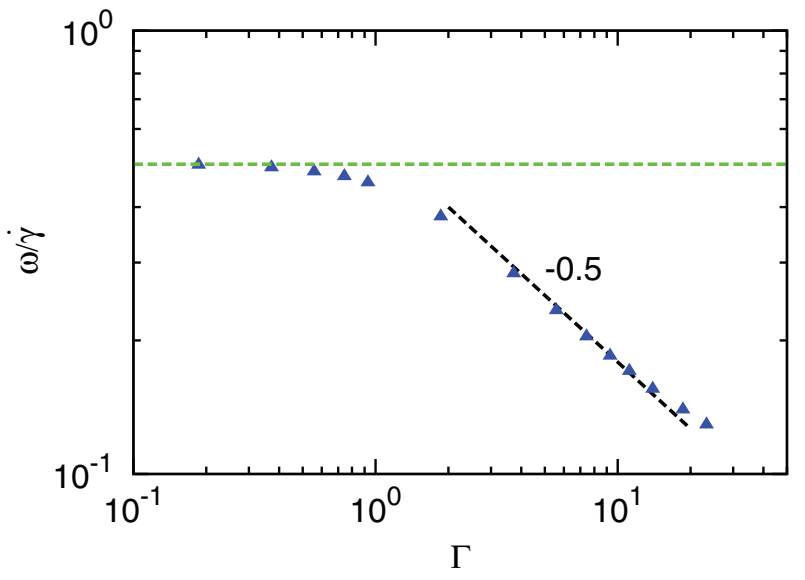

FIG. 5. Reduced rotation frequency $\omega / \dot{\gamma}$ of dumbbells as a function of reduced shear rate $\Gamma$. The other parameters are the same as those in Fig. 1. The green and black dashed lines indicate the asymptotic behavior for small and large reduced shear rates, respectively.

approximate analytical expression derived in Ref. 13. As the shear rate increases beyond $\Gamma=1$, however, $\eta_{r}$ starts to decrease quickly and then follows a power-law behavior

$$
\eta_{r} \sim \Gamma^{-\alpha}
$$

with the exponent $\alpha \approx 0.6$. Thus, the shear-thinning behavior of polymer suspensions is well reproduced by our model. The exponent $\alpha$ is well in the range reported by experiments and simulations. ${ }^{3,28,31,36,39,40}$ For example, $\alpha$ is found to be 0.52 in the experiment by Teixeira et al. ${ }^{31}$ Theoretical calculations predict the exponent $\alpha=2 / 3$ in the asymptotic limit of infinitely large shear rates. ${ }^{3,28,41}$

Theoretical studies of the FENE model by Warner ${ }^{42}$ suggest that the viscosity of FENE-dumbbell suspensions is determined by the parameter $b$. We, therefore, examine the viscosity of our FENE-dumbbell fluid with different spring constants but the same FENE parameter. As shown in Fig. 7(a), the results indeed fall onto the same master

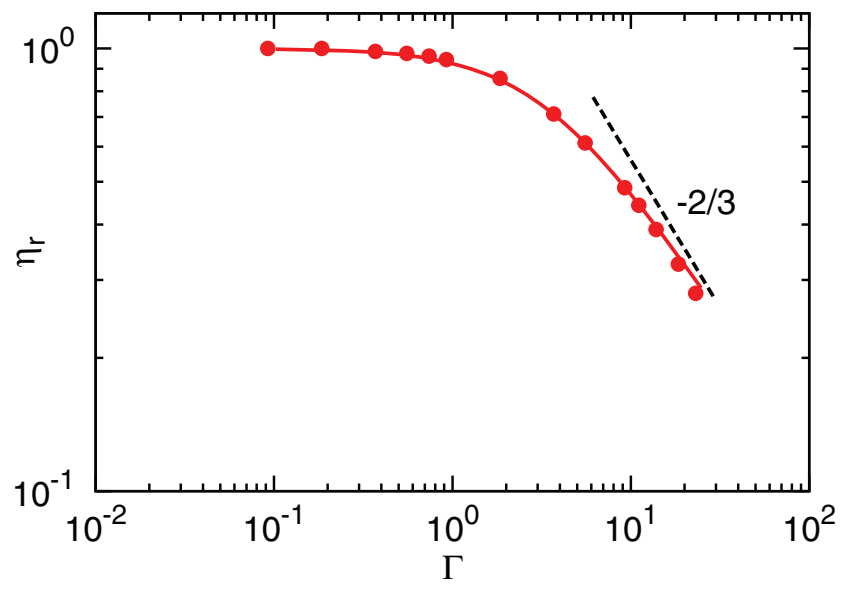

FIG. 6. The reduced viscosity $\eta_{r}$ as a function of the reduced shear rate $\Gamma$. The parameters are the same as Fig. 1. The solid line shows a fit to the Carreau-type function (18) with the parameters $\mu=0.60, q=1.38$, and $\Gamma_{0}=3.24$, the dashed line indicates the asymptotic scaling law for high Weissenberg numbers.
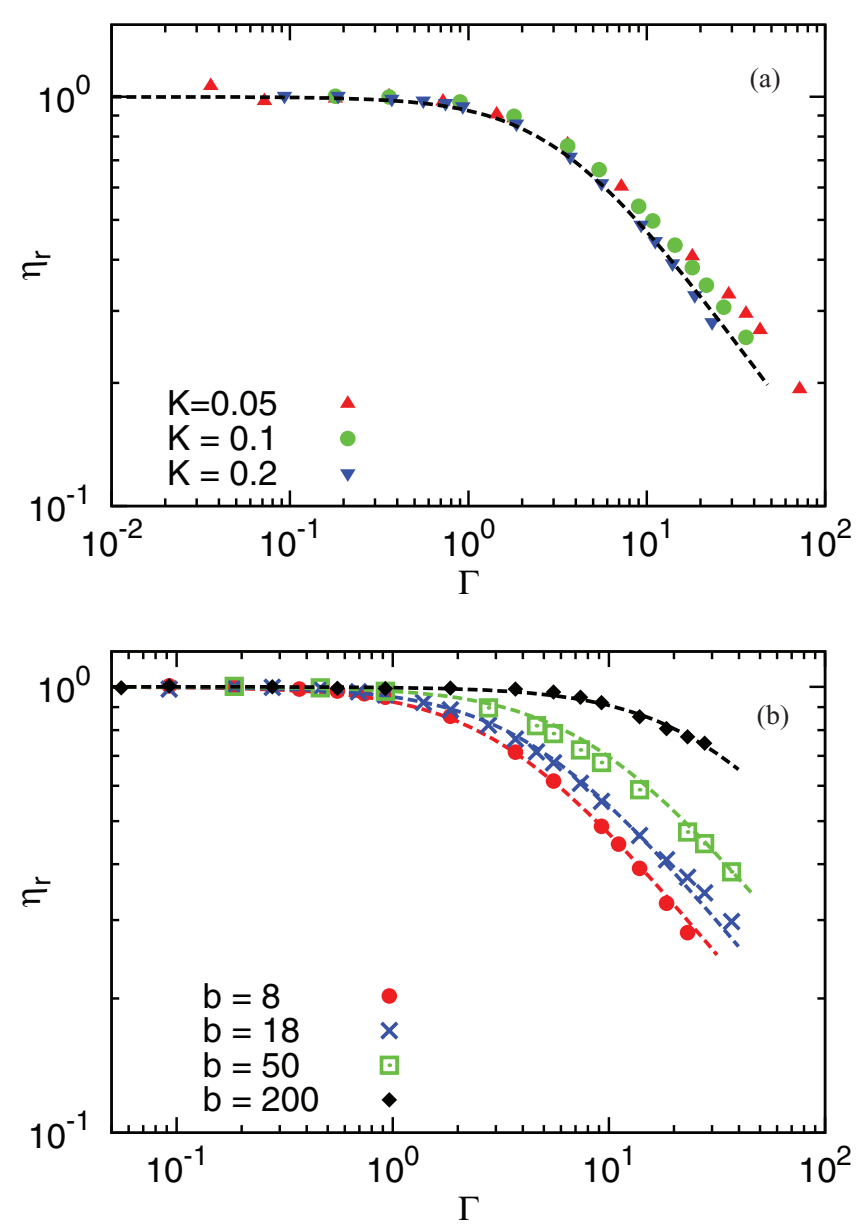

FIG. 7. The reduced viscosity $\eta_{r}$ as a function of the reduced shear rate $\Gamma$ for dumbbells with (a) same FENE parameter $b=8$ but different spring constant $k$; (b) same spring constant $k=0.2$ but different FENE parameters. Dashed lines show fits to the Carreau-type expression (18) with the parameters $\mu$ $=0.60, q=1.38$, and $\Gamma_{0}=3.24$ for $b=8, \Gamma_{0}=4.40$ for $b=18, \Gamma_{0}$ $=8.27$ for $b=50$, and $\Gamma_{0}=27.5$ for $b=200$.

curve. Simulations with different $b$ parameters show different shear-thinning behaviors over a considered range of shear rates (see Fig. 7(b)); the shear-thinning behavior becomes weaker with increasing $b$, in agreement with the predictions of Ref. 42. Theory suggests that the shear-thinning behavior will vanish as $b \rightarrow \infty$, which corresponds to harmonic dumbbells. ${ }^{42}$ In addition, our simulation results are well described by the Carreau-type expression ${ }^{39}$

$$
\eta=\eta_{0}\left[1+\left(\Gamma / \Gamma_{0}\right)^{q}\right]^{-\mu / q} .
$$

An important feature of viscoelastic fluids is that normalstress differences are non-zero, which accounts for the well-known rod climbing and extrusion swelling effects of polymers. ${ }^{2}$ However, it is very difficult to determine normalstress differences, both in experiments and simulations. We determined the first normal stress coefficient $\Psi_{1}$ (see Fig. 8). $\Psi_{1}$ is essentially independent of the shear rate in the lowflow regime $(\Gamma \leq 1)$. In the regime $\Gamma>1$, where the viscosity shows shear-thinning behavior, $\Psi_{1}$ also decreases in a powerlaw fashion as

$$
\Psi_{1} \sim \Gamma^{-\beta}
$$




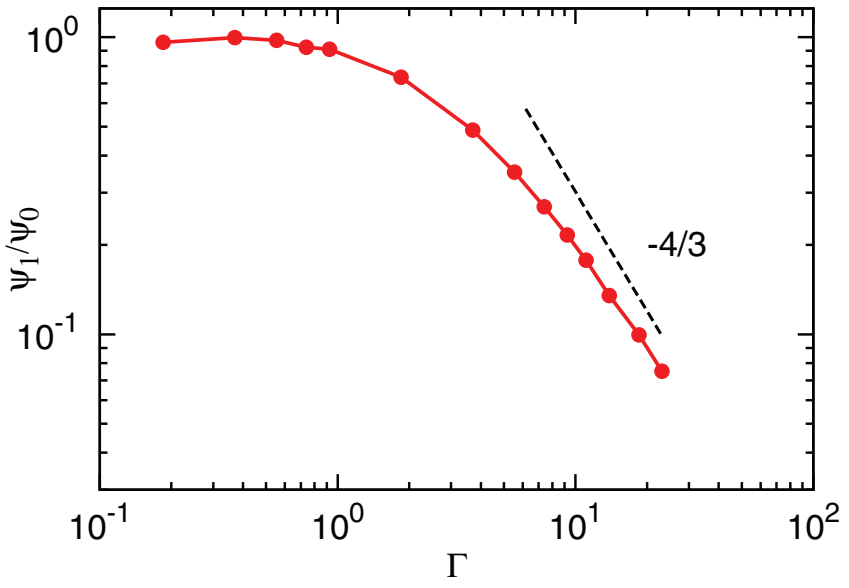

FIG. 8. The reduced coefficient of the first normal stress difference as a function of the reduced shear rate $\Gamma$. The other parameters are the same as those in Fig. 1. The solid line is a guide to the eye, the dashed line indicates the asymptotic scaling law.

Within the accuracy of the simulations, the exponent is close to $\beta \approx 4 / 3$, in agreement with theoretical predictions ${ }^{3,28}$ for polymer systems as well as a broad range of simulations as discussed in Ref. 36.

\section{FENE-dumbbell fluids in three dimensions}

The model introduced in Sec. II can easily be generalized to three spatial dimensions. In the simulations, we study a system of size $L_{x}=L_{y}=L_{z}=30$ with density of MPC particles $\rho=10$, collision time $h=0.02$, spring constant of the FENE dumbbell $k=0.2$, and FENE parameter $b=8$.

Results for the viscosity as a function of the shear rate are shown in Fig. 9. Shear thinning is found for reduced shear rates $\Gamma>1$, very similar as observed for the two-dimensional systems in Sec. III B. In fact, a more detailed comparison of the data of Figs. 6 and 9 shows that the shear-thinning

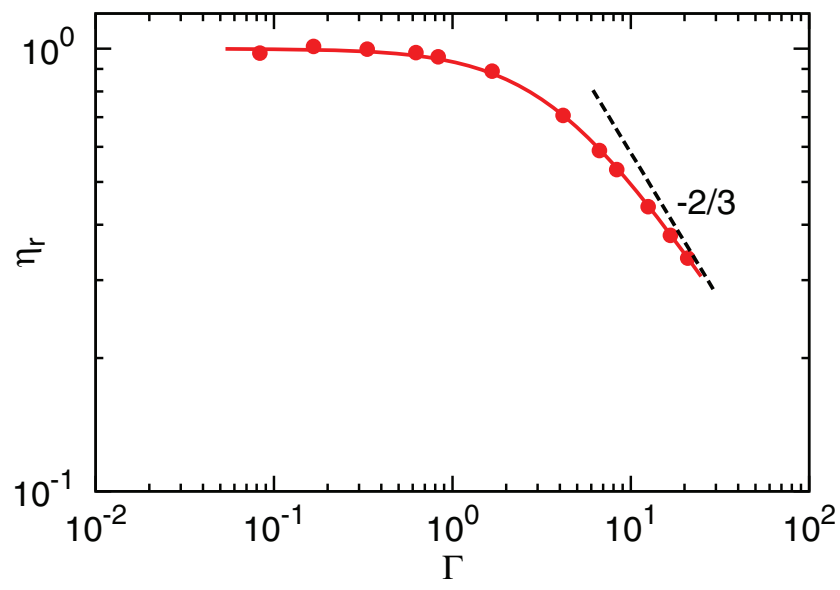

FIG. 9. The reduced viscosity $\eta_{r}$ as a function of the reduced shear rate $\Gamma$ in three dimensions. The parameters are collision time $h=0.02$, density $\rho=10$, spring constant $k=0.2$, and FENE parameter $b=8$. The solid line shows a fit to the Carreau-type function (18) with the parameters $\mu=0.60$, $q=1.38$, and $\Gamma_{0}=3.61$, the dashed line indicates the asymptotic scaling law for high Weissenberg numbers. curves are nearly the same, as can be seen from the parameter values in the Carreau fits. This similarity is not a coincidence, because hydrodynamic interactions should play a minor role only, ${ }^{36,43}$ and dumbbells are mostly aligned in the shear-gradient plane in the shear-thinning regime.

\section{COLLOID DYNAMICS UNDER SHEAR FLOW}

The results of Sec. III demonstrate that FENE-dumbbell fluids provide a good description of viscoelastic solvents. As a first application to more complex systems, we now investigate the dynamical behavior of a colloid in shear flow, by comparing Newtonian and viscoelastic solvents.

Early experiments in the 70 s of the last century indicated that there is no difference between the behavior of spherical particles suspended in Newtonian and viscoelastic fluids. ${ }^{4-46}$ It is well known that the rotation frequency $\omega_{s}$ of a sphere in a Newtonian solvent is identical to $\dot{\gamma} / 2$ in the absence of inertia, Brownian motion, and gravity, and with no-slip boundary conditions. ${ }^{38}$ However, the theoretical analysis by D'Avino et al. ${ }^{5}$ based on constitutive equations (BirdCarreau, Maxwell, Phan Thien-Tanner, and Giesekus models) for the stress tensor, suggests a reduced rotation frequency $\omega_{s} / \dot{\gamma}<1 / 2$ in a viscoelastic solvent, which is supported by recent experiments. ${ }^{6}$

In our simulation, a colloid in two dimensions is modeled as a circular ring consisting of $N_{m}=48$ monomers of mass $M=\rho$ each. Monomers are connected by the springs with the potential

$$
U_{s}=\frac{k_{s}}{2} \sum_{i}\left(\left|\boldsymbol{r}_{i+1}-\boldsymbol{r}_{i}\right|-l\right)^{2},
$$

where $l$ is the equilibrium bond length. A bending potential

$$
U_{b}=\frac{k_{b}}{2} \sum_{i}\left(\boldsymbol{r}_{i+2}-\boldsymbol{r}_{i+1}\right)\left(\boldsymbol{r}_{i+1}-\boldsymbol{r}_{i}\right)
$$

is added to favor a circular shape. The equilibrium bond length is set equal to the collision cell size $a$. The resulting circle has a radius of $R_{\text {col }}=7.64 a$. The spring constant $k_{s}$

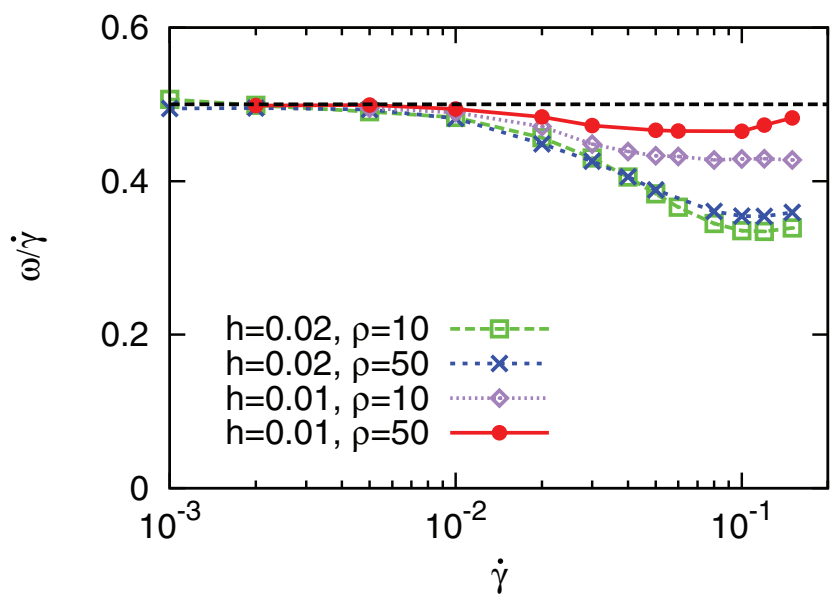

FIG. 10. Reduced rotation frequency $\omega_{s} / \dot{\gamma}$ of a colloid in a Newtonian fluid with indicated collision time $h$ and particle density $\rho$. The size of the simulation box is $L_{x}=120, L_{y}=80$. 


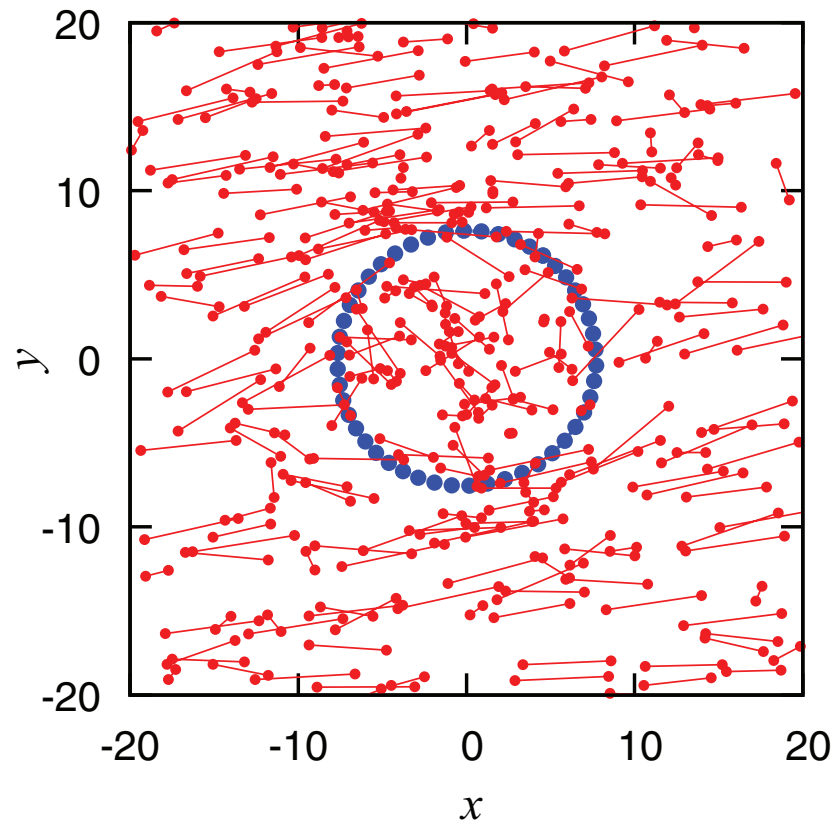

FIG. 11. Snapshot of dumbbell conformations in shear flow near a colloidal particle (blue circle). Only a fraction of $0.6 \%$ of dumbbells is shown. The reduced shear rate is $\Gamma=23.5(\dot{\gamma}=0.1)$. Note that the dumbbells in the interior of the colloid are not stretched. The parameters are collision time $h$ $=0.01$, density $\rho=50$, spring constant $k=0.2$, and FENE parameter $b=8$. The system size is $L_{x}=120, L_{y}=80$.

is chosen rather large to avoid stretching of bonds, ${ }^{47}$ and the bending rigidity $k_{b}$ is chosen such that the eccentricity of the ring is smaller than 0.1 (for all considered shear rates). To ensure a perfect circular confirmation even for high shear rates, extra harmonic springs with spring constant $k_{s}$ and equilibrium bond length $l^{\prime}=15.3$ are introduced between beads 1 and 25, 4 and 28, etc. Thus, the colloid model resembles the wheel of a bicycle.

Our motivation for using a ring with connected point particles to model the colloid instead of a hard sphere with no-slip boundary conditions is two-fold. First, this approach is technically simpler and computationally more efficient.

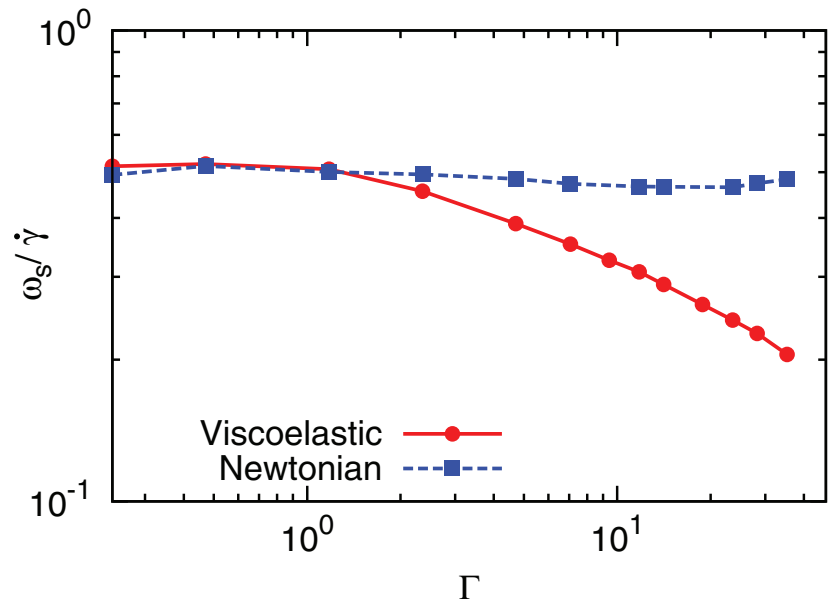

FIG. 12. Reduced rotation frequency $\omega_{s} / \dot{\gamma}$ of a colloid in a viscoelastic and Newtonian fluid, respectively. The parameters are the same as in Fig. 11.
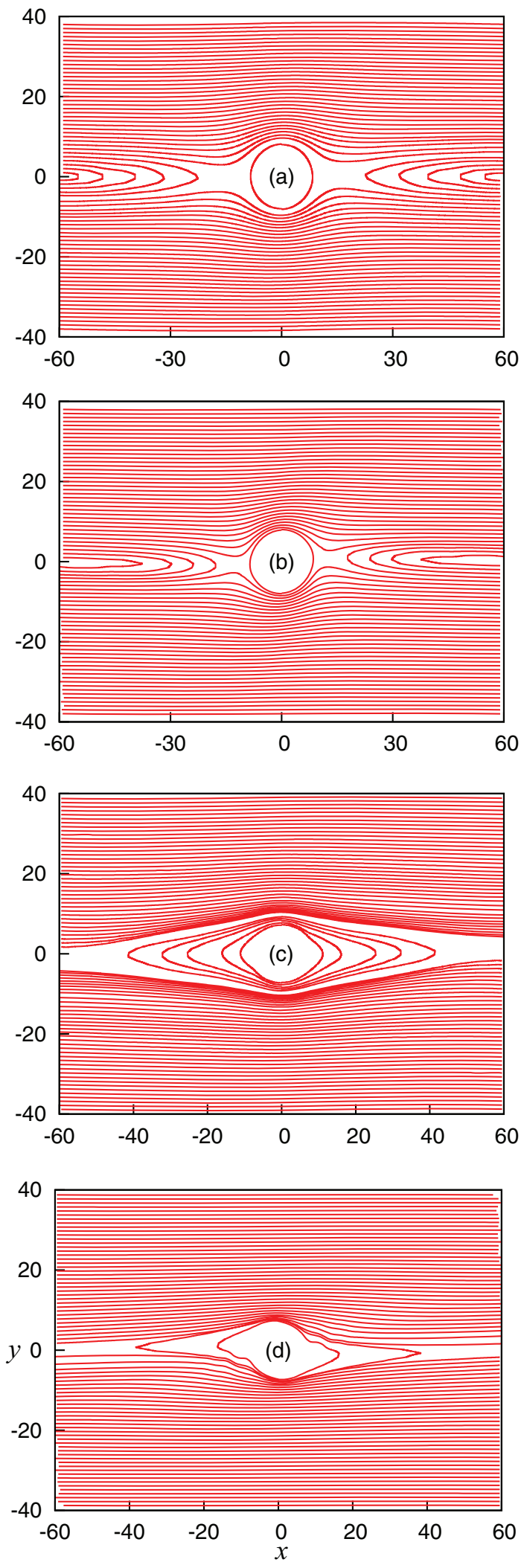

FIG. 13. Streamlines in Newtonian fluids with (a) $\dot{\gamma}=0.01$, (b) $\dot{\gamma}=0.1$, and in viscoelastic fluids with (c) $\Gamma=2.35(\dot{\gamma}=0.01$ ), (d) $\Gamma=23.5$ $(\dot{\gamma}=0.1)$. The parameters are the same as in Fig. 11.

Second, it has been shown in Ref. 13 for harmonic dumbbells that the equilibrium monomer density near a hard wall is reduced by a factor of two compared to the monomer density 
far from a wall. The same argument applies for FENE dumbbells. This density reduction might lead to artifacts such as an increased slip near the surface. In contrast, the monomer density in equilibrium is completely homogeneous for the connected point-particle model.

For the coupling of such a colloid to the MPC fluid, we adopt the well-established method for polymers, in which the monomers are included in the collision step. ${ }^{8,9,48}$ In the streaming step, the equations of motion for the monomers are integrated by the velocity Verlet algorithm, but with a smaller time step $\Delta t_{c o l}=0.0001$ than that for the FENE dumbbells because of the stiffer potentials.

We first examine the behavior of a colloid under shear flow in a Newtonian fluid. The rotation frequency of the colloid is calculated according to Eq. (15) with moment of inertia $I=N_{m} m R_{c o l}^{2}$. Results are shown in Fig. 10. In the low shearrate regime, the reduced rotation frequency is $\omega_{s} / \dot{\gamma}=0.5$, in agreement with theoretical predictions. However, in the higher shear-rate regime, $\omega / \dot{\gamma}$ drops below this value. The reduction of $\omega / \dot{\gamma}$ may be caused by inertia or by compressibility effects. The inertia effects of the fluid ${ }^{49,50}$ can be characterized by the colloidal Reynolds number $R e$

$$
R e=\frac{\dot{\gamma} R_{c o l}^{2}}{v},
$$

where $v=\eta /(m \rho)$ is the kinematic viscosity; they should be negligible for $R e \ll 1$. For the simulation results of Fig. 10, obtained with $\rho=10$ and $h=0.02$, we obtain $R e=0.32$ for $\dot{\gamma}=0.01$ and $R e=3.2$ for $\dot{\gamma}=0.1$. Evidently, the inertia effect cannot be ignored for shear rates $\dot{\gamma} \gtrsim 0.02$. The Reynolds number can be reduced at a constant shear rate by increasing the kinematic viscosity $v$. In MPC simulations, this is achieved by reducing the collision time step $h$. The compressibility of the fluid can be reduced by increasing the particle density $\rho$. Therefore, we examine systems with smaller time step $h=0.01$, or higher fluid number density $\rho=50$, or both. The effect of higher density is small, so that we can rule out compressibility effects. However, the results for smaller $h$ are close to the theoretical expectation $\omega / \dot{\gamma}=1 / 2$, see Fig. 10, i.e., inertial effects are sufficiently suppressed.

We investigate next the rotation of a colloid under shear flow in the viscoelastic solvent described in Sec. III. A snapshot of a typical dumbbell arrangement in the vicinity of the colloid is shown in Fig. 11. Results for the rotation frequency are compared in Fig. 12 with those of a Newtonian solvent. For small shear rates, $\Gamma=\dot{\gamma} \tau<1$ (with the characteristic time $\tau=235.4$ of the viscoelastic fluid for the employed parameter set), the rotation dynamics is identical and independent of the shear rate. However, for $\Gamma>1$, a significant slowing down of the rotation frequency is observed for the viscoelastic solvent (see Fig. 12), in agreement with recent experimental result. ${ }^{6}$ Since the kinematic viscosity of FENEdumbbell fluid is larger than that of a Newtonian MPC fluid, inertial effect in the viscoelastic solvent is negligible. The slowing down is thus clearly caused by viscoelastic effects.

For any $R e$, a freely suspended circular colloid in shear flow (equivalent to a cylinder in three dimensions, with the cylinder axis perpendicular to the flow-gradient plane) in a Newtonian fluid exhibits a region of closed streamlines near

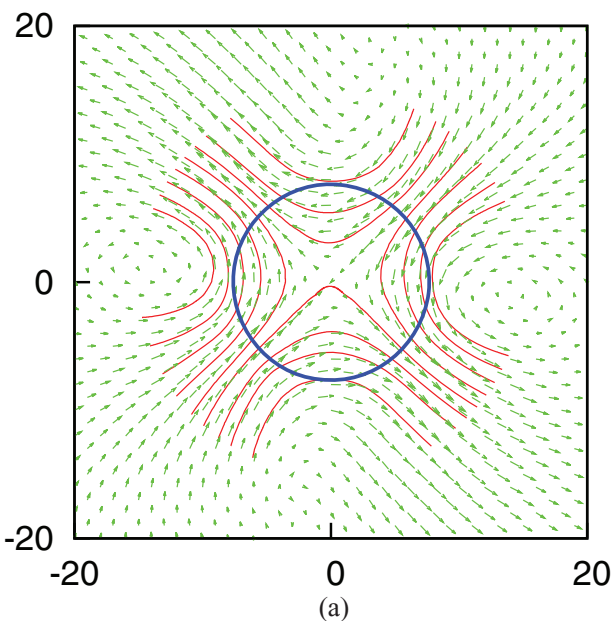

(a)

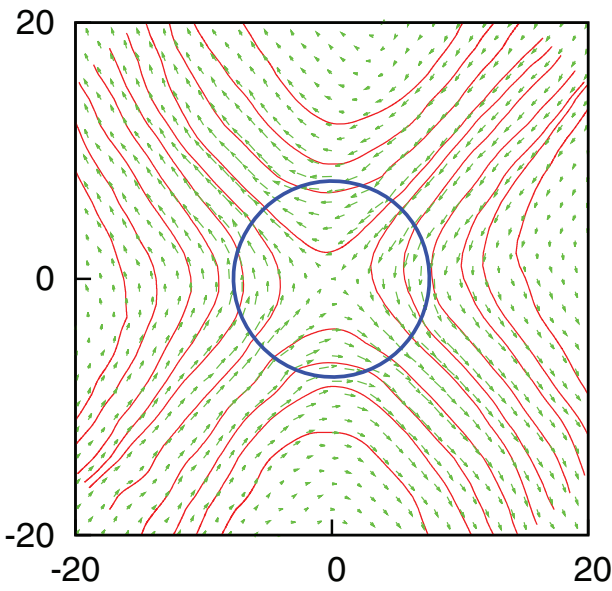

(b)

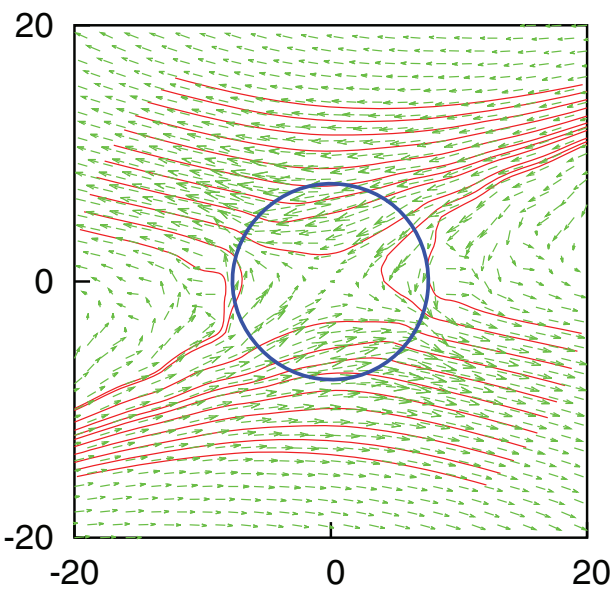

(c)

FIG. 14. Net velocity fields $\mathbf{v}-\mathbf{v}^{\text {ideal }}$ and corresponding streamlines of (a) a Newtonian fluid with shear rate $\dot{\gamma}=0.01$, (b) a viscoelastic fluid with $\Gamma=2.35(\dot{\gamma}=0.01)$, and (c) a viscoelastic fluid with $\Gamma=23.5(\dot{\gamma}=0.1)$. Parameters are $\rho=50, h=0.01$, and for the viscoelastic case $k=0.2$, and $b=8$. The blue circle indicates the position and size of the colloid.

the colloid surface. For $R e=0$ (Stokes flow), this region is known to extend to infinity in the flow direction; furthermore, the streamlines are both fore-aft and up-down symmetric. ${ }^{51}$ For $R e>0$, reverse flow zones appear near the centerline through the colloid axis, which are separated from the closed streamlines by two stagnation points (see Fig. 13(a)). The stagnation points move closer to the colloid surface and the 


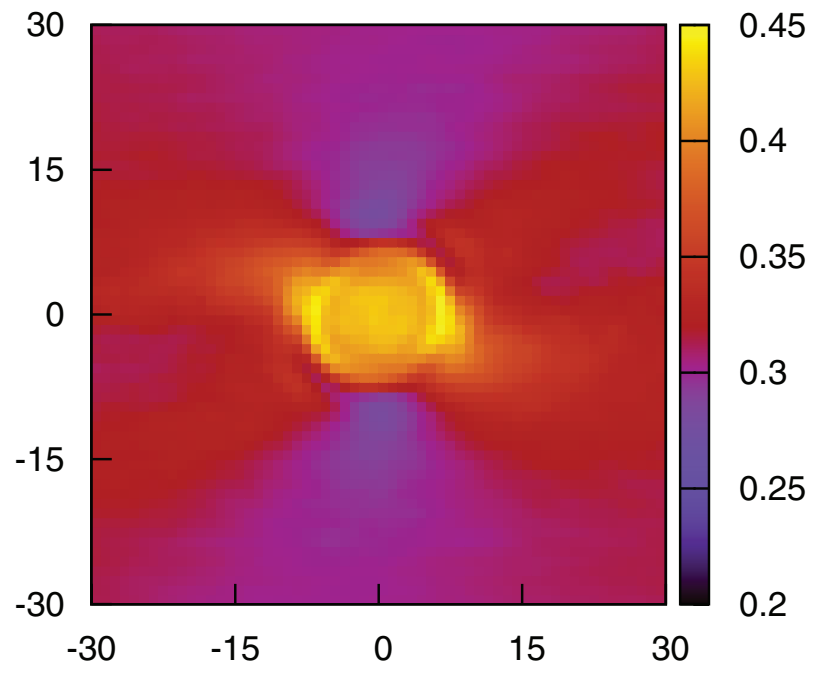

(a)

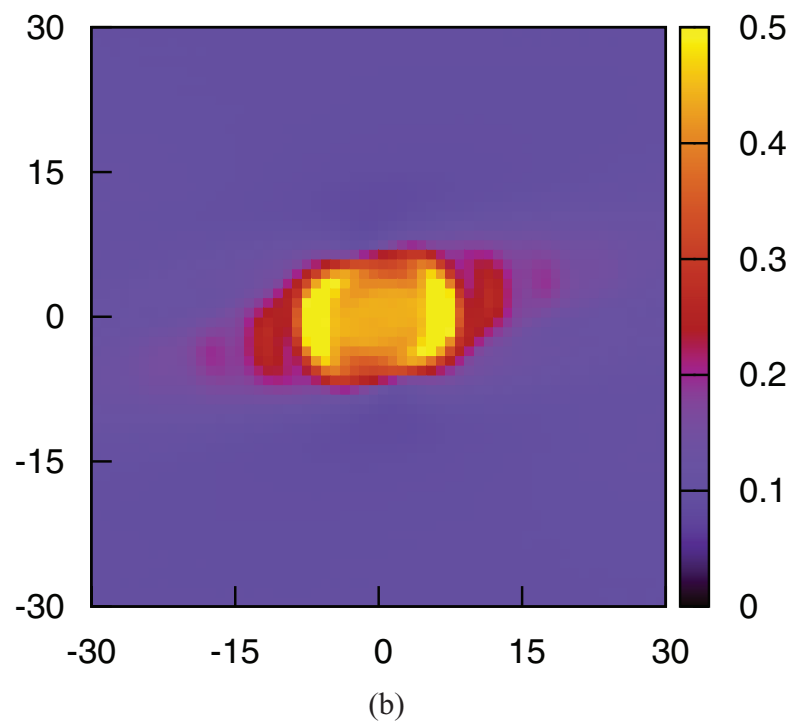

FIG. 15. Reduced rotation velocity $\omega / \dot{\gamma}$ of FENE dumbbells spatially resolved in the vicinity of a colloid in shear flow for the shear rates (a) $\Gamma=2.35$ $(\dot{\gamma}=0.01)$ and (b) $\Gamma=23.5(\dot{\gamma}=0.1)$. The other parameters are $\rho=50$, $h=0.01, k=0.2$, and $b=8$.

extension of the reverse regions increases with increasing $\operatorname{Re}$ (see Fig. 13(b)), in agreement with previous numerical results. ${ }^{50}$

In contrast, for a viscoelastic fluid the streamlines are quite different. Figure 13(c) shows streamlines of a viscoelastic fluid with the same low shear rate as that in Fig. 13(a). The extension of the inner region is much larger than that of the Newtonian fluid and the reverse regions disappear. For the higher shear rate, Fig. 13(d) shows that the shape of the inner region gets distorted and the fore-aft and up-down symmetries of the streamlines are broken.

We would like to emphasize that there is, of course, a smooth crossover from Newtonian to viscoelastic behavior depending on the relative magnitude of viscous and elastic contributions to the fluid stress tensor.

Another way to visualize the difference of the flow behavior of Newtonian and viscoelastic fluids around a circular

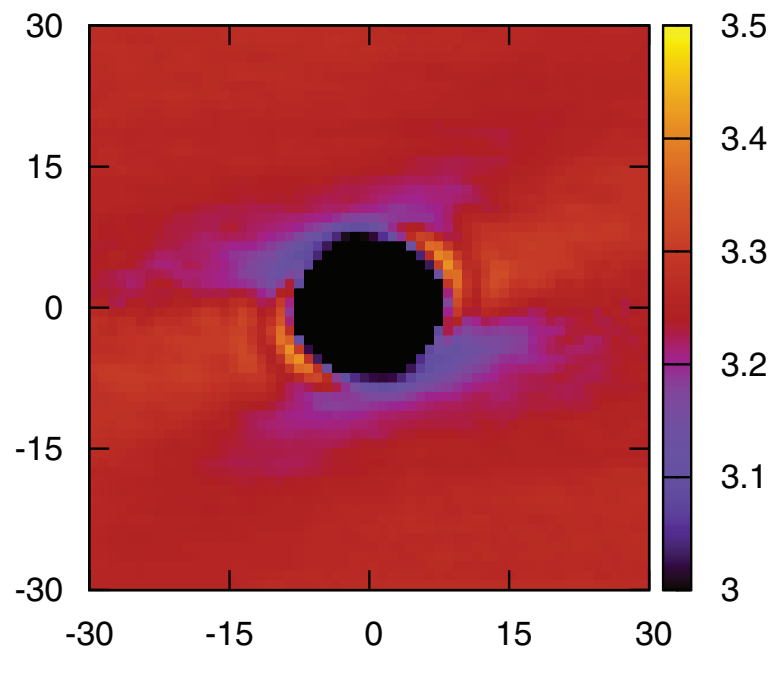

(a)

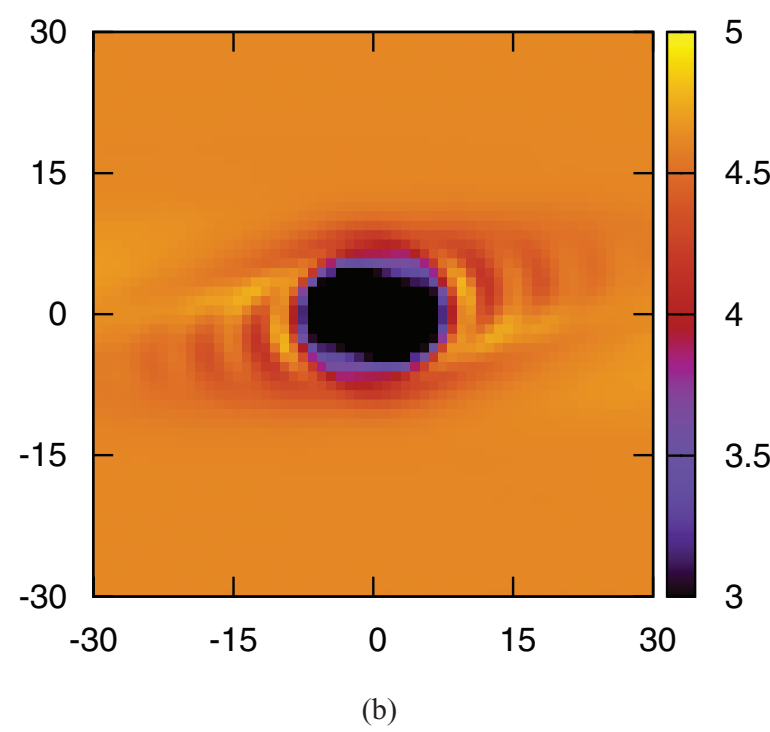

FIG. 16. Average extension $R$ of FENE dumbbells spatially resolved in the vicinity of a sphere in shear flow for the shear rates (a) $\Gamma=2.35(\dot{\gamma}=0.01)$ and (b) $\Gamma=23.5(\dot{\gamma}=0.1)$. The other parameters are $\rho=50, h=0.01, k$ $=0.2$, and $b=8$.

colloid is to consider the disturbance flow, which is obtained by subtracting the simple shear-flow field $\overline{\boldsymbol{v}}^{\text {ideal }}=\dot{\gamma}$ y $\boldsymbol{e}_{x}$ from the full velocity field $\overline{\boldsymbol{v}} \cdot{ }^{50}$ Results are shown in Fig. 14. For low-Reynolds-number Newtonian fluids, the colloid induces a flow similar in shape but opposite in direction to the extensional component of $\overline{\boldsymbol{v}}^{\text {ideal }}$, with some vorticity due to finite $\operatorname{Re}$ (see Fig. 14(a)), in agreement with previous results. ${ }^{50}$ For viscoelastic fluids, the disturbance flow for small shear rates looks similar to that of Newtonian fluids, but with suppressed vorticity (see Fig. 14(b)). For larger shear rates, the disturbance flow becomes much more parallel to the flow direction (see Fig. 14(c)).

Not only is the rotational dynamics of a circular colloid affected by the dynamics of the viscoelastic solvent, vice versa the dynamics and conformations of the dumbbells of the solvent are also affected by the presence of the colloid. We consider the local rotation velocity and extension of dumbbells in the vicinity of a colloid in shear flow. Results 
for the local rotation velocity are shown in Fig. 15. In the low shear-rate regime, there are four regions, roughly along the diagonals in the $x y$-plane (see Fig. 15(a)), where the dumbbells rotate faster than those in the bulk state. These regions extend over a distance comparable to the colloid diameter. In the high shear-rate regime, the picture looks quite different (see Fig. 15(b)). The regions with rotational velocities considerably different from that of the bulk are now much more confined to the close vicinity of the colloid, the region of higher rotation velocity is essentially on both sides of the colloid in the flow direction (see Fig. 15(b)).

The spatially resolved dumbbell extension around a colloid is shown in Fig. 16. At low shear rates, higher extension is found on the side where the flow departs from the colloid, whereas dumbbells are compressed when they flow toward the colloid. For higher shear rates, the dumbbell extension is quite uniform, with asymmetric streaks roughly parallel to the flow direction.

\section{SUMMARY AND CONCLUSIONS}

We have studied FENE-dumbbell fluids with multiparticle collision dynamics as a mesoscale hydrodynamics model for shear-thinning viscoelastic fluids. The fluid stress tensor has been calculated for a fluid in simple shear flow, and the viscosity and the first normal stress coefficient have been determined as a function of the shear rate. The shear-thinning behavior of dilute polymer solutions has been well reproduced. There are different explanations on the origin of shear thinning, such as excluded-volume interaction, the finite extensibility of polymers, or hydrodynamic interactions. ${ }^{11,52}$ The comparison of our results for FENE-dumbbell fluids and those for harmonic-dumbbell fluids of Ref. 13-in both cases with collisional interactions only-shows that shear thinning is determined by the finite dumbbell extensibility, in agreement with previous analytical results for dumbbells in dilute solution. ${ }^{2,3,28}$ The MPC simulations correctly provide the conformational properties and dynamic information of dumbbells under shear.

In contrast to the pronounced shear thinning of FENEdumbbell fluids in MPC simulations, DPD simulations of similar fluids ${ }^{14}$ show only a very weak shear-thinning behavior. A possible reason for the weak shear thinning in DPD might be that the contribution of the FENE interactions to the zeroshear viscosity is relatively small, comparable in magnitude to the viscosity of the monomer fluid (as can be inferred from the study of mixtures of normal DPD particles and dumbbells in Ref. 15). Note that we present the relative change of the shear viscosity rather than the total viscosity. Data for the total zero-shear viscosity of the harmonic-dumbbell fluid with MPC have already been presented in Ref. 13 (and are essentially identical for FENE dumbbells); these data show that the contribution of the FENE interactions in our case is about one order of magnitude larger than the contribution of the monomer fluid for spring constant $k=0.2$, and increases linearly with $1 / k$.

To demonstrate the benefit of our model for studies of more complex fluids, such as colloids suspended in a viscoelastic solvent, we have investigated the dynamic behavior of a circular colloidal particle (in two dimensions) in Newtonian and viscoelastic fluids under shear flow. In a Newtonian fluid, the rotation frequency agrees with theoretical expectations in the low-Reynolds-number regime very well. In a viscoelastic fluid, the rotation frequency is significantly reduced for shear rates $\dot{\gamma} \tau>1$, where $\tau$ is the relaxation time of the dumbbells. This result confirms the slowing down observed in a recent experiment. ${ }^{6}$ Our simulations also demonstrate that the introduction of dumbbells changes the flow field around a colloid. The extension of the region with closed streamlines around the colloid is much larger for a viscoelastic fluid than in the Newtonian case. At high shear rates, the fore-aft and up-down symmetry is broken. Furthermore, the simulations show that the extension and rotation frequency of the dumbbells acquires a spatial dependence near the colloid.

In conclusion, we have shown that the FENE-dumbbell fluid in combination with the multiparticle collision dynamics technique provides a very promising approach to study the behavior of colloids, polymers, or membranes in shearthinning viscoelastic solvents.

\section{ACKNOWLEDGMENTS}

We thank Jan Vermant (Leuven), Ingo O. Götze, and Chien-Cheng Huang (Jülich) for stimulating discussions. Financial support by the EU FP7 Collaborative Research Project "Nanodirect" (NMP4-SL-2008-213948) is gratefully acknowledged.

${ }^{1}$ R. G. Larson, The Structure and Rheology of Complex Fluids (Oxford University Press, New York, 1999).

${ }^{2}$ R. B. Bird, R. C. Armstrong, and O. Hassager, Dynamics of Polymeric Liquids (Wiley, New York, 1998), Vol. 1.

${ }^{3}$ R. B. Bird, C. F. Curtiss, R. C. Armstrong, and O. Hassager, Dynamics of Polymeric Liquids (Wiley, New York, 1987)

${ }^{4}$ M. K. Lyon, D. W. Mead, R. E. Elliott, and L. G. Leal, J. Rheol. 45, 881 (2001).

${ }^{5}$ G. D'Avino, M. A. Hulsen, F. Snijkers, J. Vermant, F. Greco, and P. L. Maffettone, J. Rheol. 52, 1331 (2008).

${ }^{6}$ F. Snijkers, G. D'Avino, P. L. Maffettone, F. Greco, M. Hulsen, and J. Vermant, J. Rheol. 53, 459 (2009).

${ }^{7}$ R. Pasquino, F. Snijkers, N. Grizzuti, and J. Vermant, Langmuir 26, 3016 (2010).

${ }^{8}$ R. Kapral, Adv. Chem. Phys. 140, 89 (2008).

${ }^{9}$ G. Gompper, T. Ihle, D. M. Kroll, and R. G. Winkler, Adv. Polym. Sci. 221, 1 (2009).

${ }^{10}$ A. Malevanets and R. Kapral, J. Chem. Phys. 110, 8605 (1999).

${ }^{11}$ J. W. Rudisill and P. T. Cummings, J. Non-Newtonian Fluid Mech. 41, 275 (1992).

${ }^{12}$ M. Kröger, Phys. Rep. 390, 453 (2004).

${ }^{13}$ Y. G. Tao, I. O. Götze, and G. Gompper, J. Chem. Phys. 128, 144902 (2008).

${ }^{14}$ E. Somfai, A. Morozov, and W. van Saarloos, Physica A 362, 93 (2006).

${ }^{15}$ E. Sultan, J.-W. van de Meent, E. Somfai, A. N. Morozov, and W. van Saarloos, EPL 90, 64002 (2010).

${ }^{16}$ E. Allahyarov and G. Gompper, Phys. Rev. E 66, 036702 (2002).

${ }^{17}$ H. Noguchi, N. Kikuchi, and G. Gompper, EPL 78, 10005 (2007).

${ }^{18}$ I. O. Götze, H. Noguchi, and G. Gompper, Phys. Rev. E 76, 046705 (2007).

${ }^{19}$ T. Ihle and D. M. Kroll, Phys. Rev. E 63, 020201 (2001).

${ }^{20}$ T. Ihle and D. M. Kroll, Phys. Rev. E 67, 066705 (2003).

${ }^{21}$ M. Ripoll, K. Mussawisade, R. G. Winkler, and G. Gompper, Europhys. Lett. 68, 106 (2004).

${ }^{22}$ J. T. Padding and A. A. Louis, Phys. Rev. E 74, 031402 (2006).

${ }^{23}$ H. Noguchi and G. Gompper, Phys. Rev. E 78, 016706 (2008). 
${ }^{24}$ M. P. Allen and D. J. Tildesley, Computer Simulation of Liquids (Clarendon, Oxford, 1987).

${ }^{25}$ R. G. Winkler and C. C. Huang, J. Chem. Phys. 130, 074907 (2009).

${ }^{26}$ N. Kikuchi, C. M. Pooley, J. F. Ryder, and J. M. Yeomans, J. Chem. Phys. 119, 6388 (2003).

${ }^{27}$ T. Ihle, E. Tüzel, and D. M. Kroll, Phys. Rev. E 72, 046707 (2005).

${ }^{28}$ R. G. Winkler, J. Chem. Phys. 133, 164905 (2010).

${ }^{29}$ D. E. Smith, H. P. Babcock, and S. Chu, Science 283, 1724 (1999).

${ }^{30}$ C. M. Schroeder, R. E. Teixeira, E. S.G. Shaqfeh, and S. Chu, Phys. Rev. Lett. 95, 018301 (2005).

${ }^{31}$ R. E. Teixeira, H. P. Babcock, E. S.G. Shaqfeh, and S. Chu, Macromolecules 38, 581 (2005).

${ }^{32}$ S. Gerashchenko and V. Steinberg, Phys. Rev. Lett. 96, 038304 (2006).

${ }^{33}$ P. S. Doyle, B. Ladoux, and J.-L. Viovy, Phys. Rev. Lett. 84, 4769 (2000).

${ }^{34}$ R. G. Winkler, Phys. Rev. Lett. 97, 128301 (2006).

${ }^{35}$ Y. Zhang, A. Donev, T. Weisgraber, B. J. Alder, M. G. Graham, and J. J. de Pablo, J. Chem. Phys. 130, 234902 (2009).

${ }^{36}$ C.-C. Huang, G. Sutmann, G. Gompper, and R. G. Winkler, EPL 93, 54004 (2011).
${ }^{37}$ C. Aust, S. Hess, and M. Kröger, Macromolecules 35, 8621 (2002).

${ }^{38}$ A. Einstein, Ann. Phys. 19, 289 (1906).

${ }^{39}$ C. Aust, M. Kröger, and S. Hess, Macromolecules 32, 5660 (1999).

${ }^{40}$ G. Q. Xu, J. D. Ding, and Y. L. Yang, Polymer 41, 3289 (2000).

${ }^{41}$ R. B. Bird, P. J. Dotson, and N. L. Johnson, J. Non-Newtonian Fluid Mech. 7, 213 (1980).

${ }^{42}$ H. R. Warner, Ind. Eng. Chem. Fundam. 11, 379 (1972).

${ }^{43}$ C.-C. Huang, R. G. Winkler, G. Sutmann, and G. Gompper, Macromolecules 43, 10107 (2010).

${ }^{44}$ E. Bartram, H. L. Goldsmith, and S. G. Mason, Rheol. Acta 14, 776 (1975).

${ }^{45}$ F. Gauthier, Trans. Soc. Rheol. 15, 297 (1971).

${ }^{46} \mathrm{~F}$. Gauthier, Rheol. Acta 10, 344 (1971).

${ }^{47}$ R. G. Winkler, K. Mussawisade, M. Ripoll, and G. Gompper, J. Phys. Condens. Matter 16, S3941 (2004).

${ }^{48}$ A. Malevanets and J. M. Yeomans, Europhys. Lett. 52, 231 (2000).

${ }^{49}$ C. M. Zettner and M. Yoda, Exp. Fluids 30, 346 (2001).

${ }^{50}$ D. R. Mikulencak and J. F. Morris, J. Fluid Mech. 520, 215 (2004).

${ }^{51}$ C. A. Kossack and A. Acrivos, J. Fluid Mech. 66, 353 (1974).

${ }^{52}$ J. F. Ryder and J. M. Yeomans, J. Chem. Phys. 125, 194906 (2006). 